\title{
Photofunctionalised Ti6Al4V implants enhance early phase osseointegration
}

\section{R. Yamauchi, T. Itabashi, K. Wada, T. Tanaka, G. Kumagai, Y. Ishibashi}

Hirosaki University Graduate School of Medicine, Aomori, Japan

\footnotetext{
- R. Yamauchi, MD,

- K. Wada, MD, PhD

- T. Tanaka, MD, PhD,

- G. Kumagai, MD, PhD,

- Y. Ishibashi, MD, PhD,

Department of Orthopaedic

Surgery, Hirosaki University

Graduate School of Medicine,

5 Zaifu-cho, Hirosaki, 036-8562

Aomori, Japan.

- T. Itabashi, MD, PhD,

Department of Surgery, Kuroishi

General Hospital, 1 Chome-70

Kitamicho, Kuroishi, Aomori

Prefecture 036-0541, Japan.

Correspondence should be sent to R. Yamauchi; email: ryoooota@ hirosaki-u.ac.jp

doi: $10.1302 / 2046-3758.65 . B \mid R-$ 2016-0221.R1
}

Bone Joint Res 2017;6:331-336. Received: 18 August 2016; Accepted: 31 January 2017

\section{Objectives}

Ultraviolet (UV) light-mediated photofunctionalisation is known to improve osseointegration of pure titanium (Ti). However, histological examination of titanium alloy (Ti6Al4V), which is frequently applied in orthopaedic and dental surgery, has not yet been performed. This study examined the osseointegration of photofunctionalised Ti6Al4V implants.

\section{Methods}

Ti and Ti6AI4V implants were treated with UV light, and the chemical composition and contact angle on the surfaces were evaluated to confirm photofunctionalisation. The implants were inserted into femurs in rats, and the rats were killed two or four weeks after the surgery. For histomorphometric analysis, both the bone-implant contact (BIC) ratio and the bone volume (BV) ratio were calculated from histological analysis and microcomputed tomography data.

\section{Results}

The amount of carbon and the contact angle on both implants were significantly reduced after UV irradiation. The BIC ratios for both UV light-treated implants significantly increased at two weeks, but there was no significant difference at four weeks. There was no significant difference in the BV ratios between the UV light-treated and control implants at two or four weeks.

\section{Conclusions}

This study suggests that photofunctionalisation of Ti6Al4V implants, similar to that of $\mathrm{Ti}$ implants, may promotes osseointegration in early but not in the late phase of osseointegration.

Cite this article: Bone Joint Res 2017;6:331-336

Keywords: Ultraviolet, Osseointegration, Titanium alloy, Bone-implant contact, Hydrophilicity

\section{Article focus}

- To investigate the effect of ultraviolet light-mediated photofunctionalisation on Ti6Al4V implants

\section{Key messages}

- Photofunctionalisation reduces the amount of carbon on the Ti6Al4V surface

- The surface of UV-treated Ti6AI4V implants enhances establishment of osseointegration in the early healing stage, but not in the late stage.

\section{Strengths and limitations}

- This study indicated the effect of photofunctionalisation on Ti6Al4V surface for the first time.
- Implant biomechanical tests were not performed, and the number of specimens in each group was small

\section{Introduction}

Pure titanium (Ti) and titanium alloy (Ti6Al4V) implants are often used as prosthetic devices in orthopaedic and dental surgery because of their advantages, which include corrosion resistance and good biocompatibility. ${ }^{1}$ The stiffness of Ti6Al4V is higher than that of Ti, and orthopaedic implants are often composed of the former because of the high degree of weight-bearing required from orthopaedic implants. ${ }^{2}$ The ageing population is increasing in size and the numbers of compromised hosts due to osteoporosis, dialysis, and the use of 
steroids have increased; in turn, the resulting poor integration between the bone and implant surface often causes severe complications. Therefore, it is important to minimise the time required for early and strong osseointegration.

Implant anchorage is successful when the bone is deposited directly onto the Ti surface without the intervention of soft or connective tissue. ${ }^{3}$ Early and strong establishment of osseointegration is of great clinical interest and benefit, but the total bone-implant contact (BIC) ratio remains at $50 \%$ to $65 \%$, which is far below the ideal of $100 \% .{ }^{4} \mathrm{~A}$ reduced $\mathrm{BIC}$ ratio is reportedly caused by biological ageing secondary to time-dependent biological degradation of the Ti surface. ${ }^{5} \mathrm{Ti}$ surfaces constantly absorb hydrocarbons from the atmosphere, and water and cleaning solutions after the implants are manufactured. ${ }^{6}$ Some studies have reported that the initial affinity for osteoblasts and the amount of bone- $\mathrm{Ti}$ integration are influenced by the amount of carbon on the surface. ${ }^{5,7,8}$ Additionally, the absorption of hydrocarbons leads to an increase in hydrophobicity on the implant surface. Many studies have reported that surface wettability is an important property for cell behaviour and that cell attachment onto hydrophobic surfaces tends to be weaker than that onto hydrophilic surfaces. ${ }^{9-11}$

Aita et $\mathrm{al}^{3}$ reported the use of ultraviolet (UV) lightmediated photofunctionalisation of Ti surfaces to overcome biological ageing. This innovative technology enhanced osseointegration, and the $\mathrm{BIC}$ ratio of photofunctionalised implants was increased to a near maximum level of $98.2 \%$ in their rat model. The effect of photofunctionalisation on Ti6Al4V surfaces was demonstrated in vitro and enhanced both bioactivity and osteoconductivity. ${ }^{13}$ Photofunctionalised $\mathrm{Ti}$ implants are already being used in dentistry, and clinical outcome studies have shown that the use of photofunctionalisation results in a high success rate and decreased healing time. ${ }^{12}$ Currently used dental and orthopaedic Ti implants were developed based on the concept of bone-implant integration. ${ }^{3}$ However, in vivo bone histomorphometric parameters, such as the BIC ratio on photofunctionalised Ti6Al4V, remain unclear. The purpose of this study was to examine the effect of UV light-mediated photofunctionalisation on Ti6Al4V implants.

\section{Materials and Methods}

Photofunctionalisation of Ti and Ti6Al4V implants. The implants used in this study were made from pure $\mathrm{Ti}$ and Ti6Al4V (diameter $2 \mathrm{~mm}$, length $15 \mathrm{~mm}$; the average values of surface roughness were 0.66 and 0.34 , respectively), and provided by B. Braun Aesculap Japan Co., Ltd (Tokyo, Japan). Half of the implants were treated with UV irradiation for 15 minutes using a photo device (TheraBeam Affinity; Ushio Inc., Tokyo, Japan) at an intensity of $3 \mathrm{~mW} /$ $\mathrm{cm}^{2}$. The light source mounted in the TheraBeam Affinity is a low-pressure mercury $(\mathrm{Hg})$ lamp, which emits predominantly $254 \mathrm{~nm}$ UV light. The implants were divided into

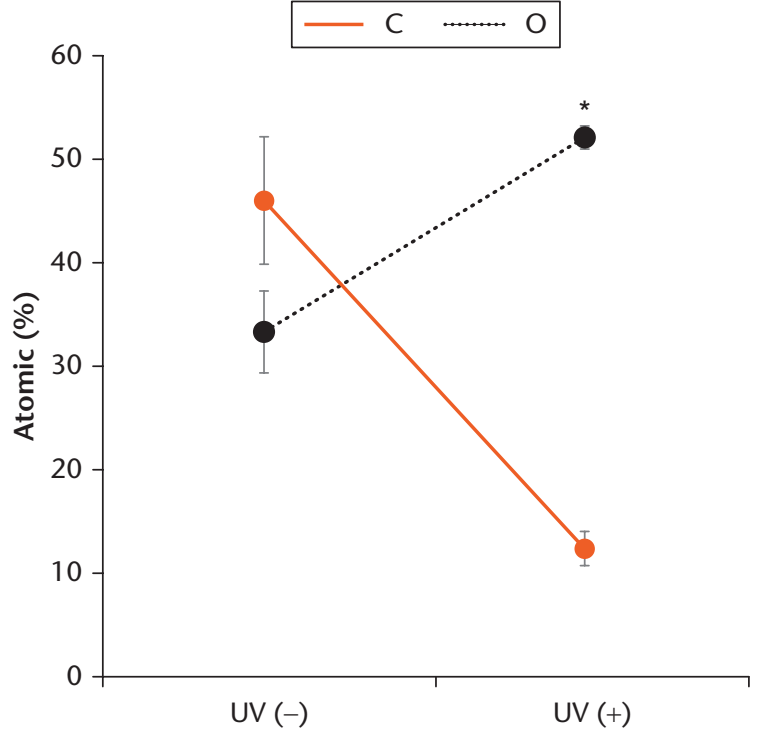

Fig. 1a

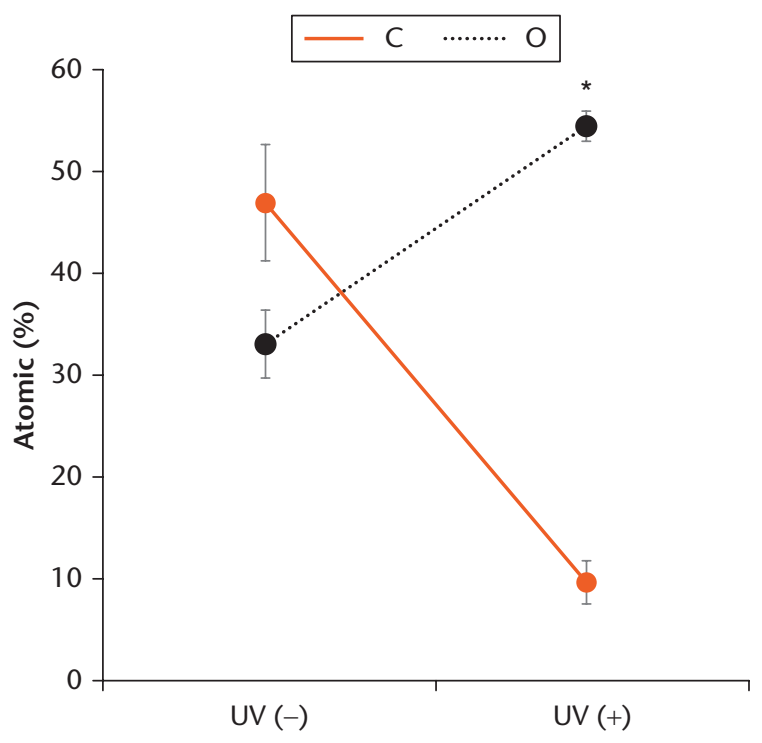

Fig. 1b

Atomic percentage on the Ti and Ti6Al4V surfaces as evaluated by XPS. (A, B) On the Ti and Ti6Al4V surfaces, the amount of carbon (C) was significantly reduced and the amount of oxygen $(\mathrm{O})$ had significantly increased after UV irradiation. ${ }^{*} p<0.05$, statistically significant difference between before and after UV irradiation $(n=5)$.

the following four groups of five: pure Ti implants without UV irradiation (Ti(-)); pure Ti implants with UV irradiation (Ti(+)); Ti6Al4V without UV irradiation (64Ti(-)); and Ti6Al4V with UV irradiation (64Ti(+)).

X-ray photoelectron spectroscopy. To confirm the removal of carbon from the implant surfaces after UV irradiation, the chemical composition of the four groups was evaluated by electron spectroscopy for chemical analysis. This analysis was performed by X-ray photoelectron spectroscopy (XPS) (PHI Quantera SXM; ULVACPHI, Inc., Kanagawa, Japan). The analysis spot, analysed 
$\mathrm{Ti}$

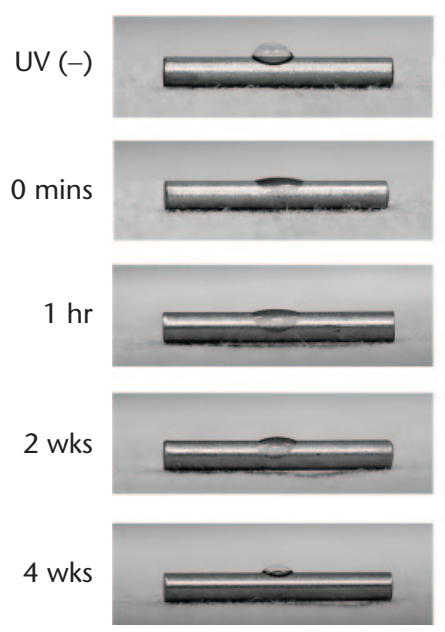

Ti6Al4V
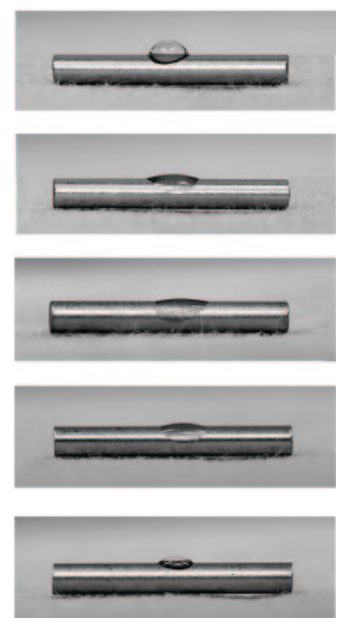

Fig. 2a

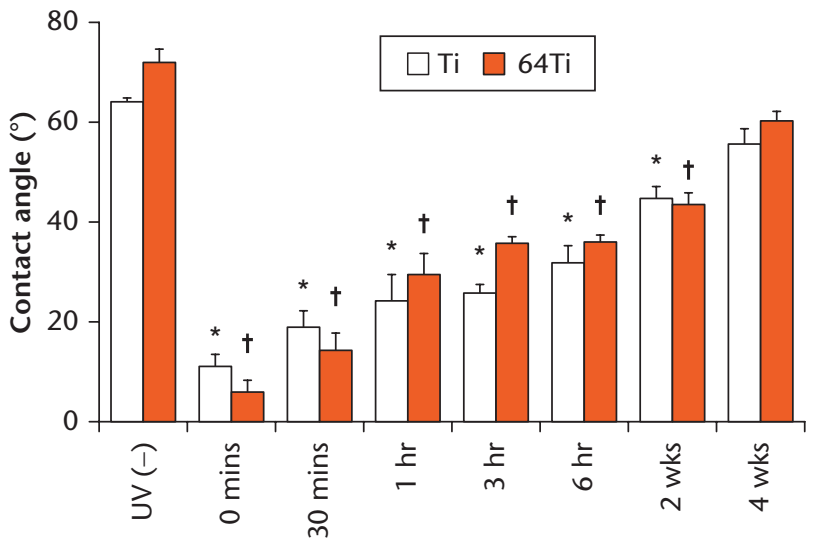

Fig. $2 b$

Hydrophilicity changed after UV irradiation as evaluated by measurement of the contact angle. a) Photographic images showed that a drop of water was deposited onto the Ti and Ti6Al4V surfaces. b) The contact angle of the $\mathrm{Ti}$ and Ti6AI4V surfaces exhibited hydrophobicity before UV irradiation and then exhibited hydrophilicity after UV irradiation. The contact angle of both surfaces showed an age-dependent reduction in hydrophilicity. ${ }^{*} p<0.05$ (paired t-test), * $\mathrm{Ti}$ immediately vs 0 minutes, 30 minutes, 1 hour, 3 hours, 6 hours, and 2 weeks after UV irradiation; †Ti6Al4V or immediately vs 0 and 30 minutes, one, three and six hours and two weeks after UV irradiation $(n=3)$.

at $7.5 \mathrm{~mm}$, was the centre of the implants, and the spot size was $300 \mu \mathrm{m}$.

Contact angle analysis. To examine the change in wettability on the implant surfaces after UV irradiation, the hydrophilicity and hydrophobicity were evaluated by measuring the contact angle with $1 \mu \mathrm{L}$ of water at room temperature immediately, and at 0 minutes, 30 minutes, one hour, three hours, six hours, two weeks, and four weeks after UV irradiation. The measurements were performed on the surfaces by the sessile drop technique, taken from the $\theta / 2$ method. ${ }^{14}$ After a drop of water was placed on the implant surface, the height (a) and contact diameter (b) of the drop were calculated by image analysis software (Image) v.1.48; National Institutes of

Health, Bethesda, Maryland). Using these measurements, the contact angle $(\theta)$ was calculated with the following formula: ${ }^{14}$

$$
\theta=2 \tan ^{-1}(2 a / b)
$$

The surface shows hydrophilicity when the contact angle is low.

Surgery. The study protocol was approved by the Animal Research Committee of Hirosaki University, and all experimentation was performed in accordance with the Rules for Animal Experimentation of Hirosaki University. Eight-week-old male Sprague-Dawley rats were used for the animal experiments. The rats were anaesthetised with $1 \%$ to $2 \%$ isoflurane. Both hind limbs were shaved, and the skin and fascia layers were opened separately. The flat aspects of the distal femurs were exposed and selected for implantation. The right and left distal femurs were drilled using a $2 \mathrm{~mm}$-diameter drill. The implants with UV irradiation were inserted into the right femur holes, and the implants without UV irradiation were inserted into the left femur holes. After implant placement, the skin and fascia were closed.

Histological analysis. Two or four weeks after the surgery, the rats were killed by intraperitoneal injection of pentobarbital, and the femurs were harvested. As in previous studies ${ }^{15}$ the specimens were embedded in methyl methacrylate without decalcification. Embedded specimens were cut perpendicular to the short axis of the implants using a microtome. Each section was stained with Villanueva-Goldner (Figs $3 \mathrm{a}$ to $3 \mathrm{~h}$ and $4 \mathrm{a}$ to $4 \mathrm{~h}$ ) and observed by light microscopy (BZ-X700; Keyence Corp., Osaka, Japan). In each histological slice, the BIC ratio for each group was calculated by the digital image analysis software (ImageJ v.1.48). The BIC ratio was calculated as the length of bone in direct contact with the surface of the implant divided by the total length of the implant, multiplied by 100 (\%). The bone in direct contact was defined as the interface at which bone tissue was located within $20 \mathrm{~mm}$ of the implant surface without intervention of soft tissue.

Bone formation around implants. The specimens were fixed in $10 \%$ buffered formalin and analysed using microcomputed tomography. Three-dimensional bone morphometric analysis was performed (Fig. 5b). The bone volume (BV) ratio was defined as the ratio of the mineralised bone volume within $100 \mu \mathrm{m}$ from the implant surface. The BV ratio was calculated as the bone occupancy in the area of interest divided by the total area of interest, multiplied by $100 \%$.

Statistical analysis. A paired $t$-test was performed to determine differences in XPS findings and in the contact angle between the UV(-) group and UV(+) group. Oneway analysis of variance with Tukey's post hoc test was performed to determine the differences in BIC ratios and BV ratios. Statistical analyses were performed using SPSS 


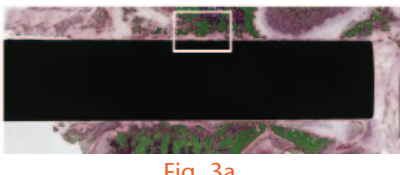

Fig. 3a

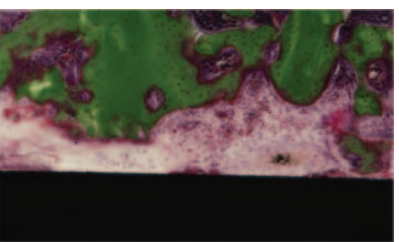

Fig. 3c

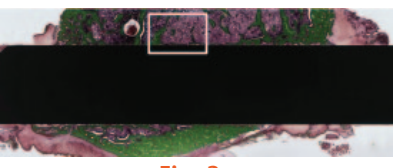

Fig. $3 e$

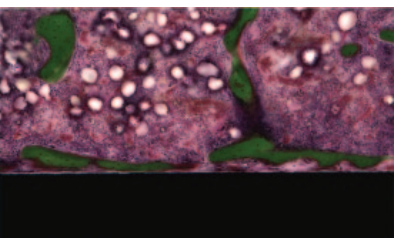

Fig. $3 g$

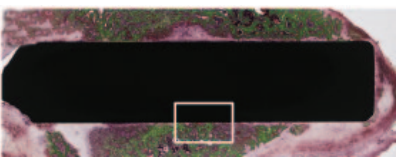

Fig. 3b

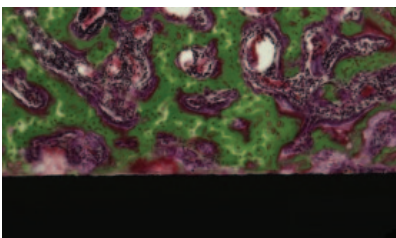

Fig. 3d

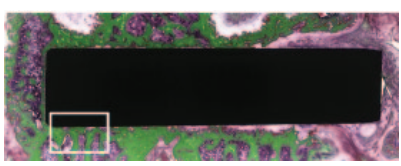

Fig. $3 f$

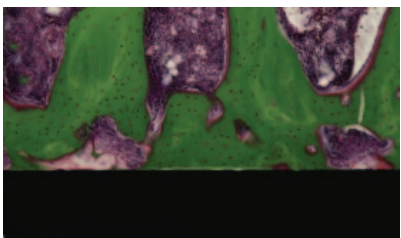

Fig. $3 \mathrm{~h}$

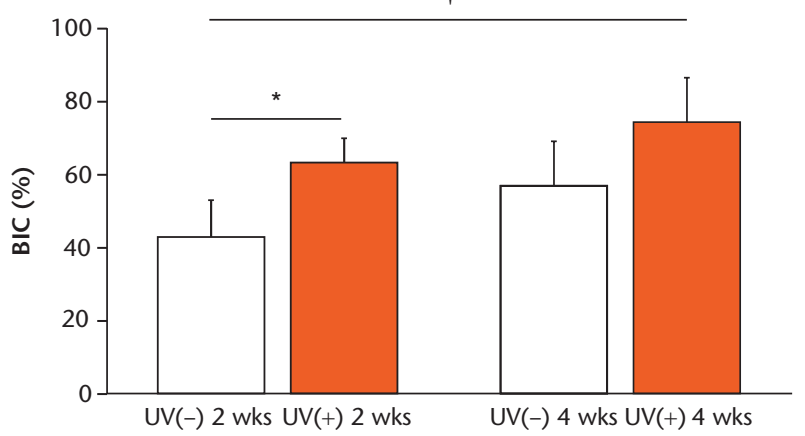

Fig. 3i

Light microscope images (magnification $\times 4$ and $\times 10$, higher magnification images of the boxed areas) two and four weeks after the implantation. The micrographs show the bone response for the $(A, C, E, G) T i(-)$ and $(B, D, F, H)$ $\mathrm{Ti}(+)$ implants. Scale bar: $200 \mu \mathrm{m}$. (I) BIC ratios for $\mathrm{Ti}(-)$ and $\mathrm{Ti}(+)$ were calculated. Results are shown as mean percentage \pm standard deviation. $p<0.05$, * $\mathrm{Ti}(-)$ vs $\mathrm{Ti}(+),{ }^{* *} 2$ vs $4 \mathrm{w}, \dagger \mathrm{Ti}(-) 2 \mathrm{w}$ vs $\mathrm{Ti}(+) 4 \mathrm{w}(\mathrm{n}=5)$.

version 12.0J (SPSS Inc., Chicago, Illinois), and p values of $<0.05$ were considered significant.

\section{Results}

X-ray photoelectron spectroscopy. In the XPS analysis, the surface of $\mathrm{Ti}$ had mean atomic percentage values of 46.4\% for carbon and $33.4 \%$ for oxygen before UV irradiation, and $12.0 \%$ for carbon and $52.6 \%$ for oxygen after UV irradiation. The surface of Ti6Al4V had mean values of $46.8 \%$ for carbon and $33.4 \%$ for oxygen before UV irradiation, and $9.2 \%$ for carbon and $52.6 \%$ for oxygen after UV irradiation. The amount of carbon on the Ti and Ti6Al4V surfaces was significantly reduced after UV

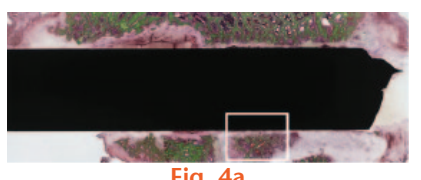

Fig. 4a

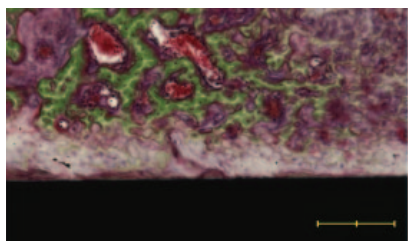

Fig. 4c

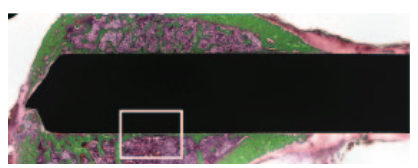

Fig. $4 \mathrm{e}$

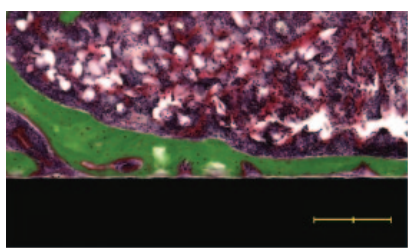

Fig. 4g

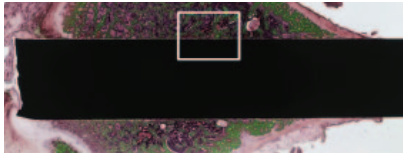

Fig. 4b

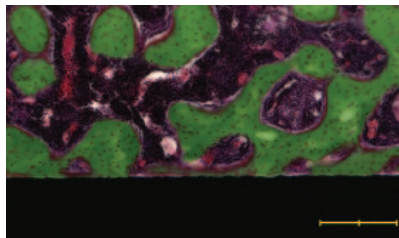

Fig. 4d

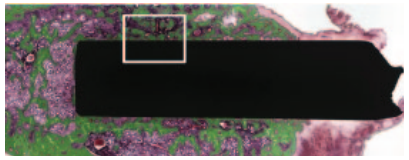

Fig. 4f

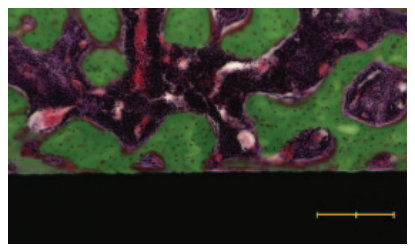

Fig. 4h

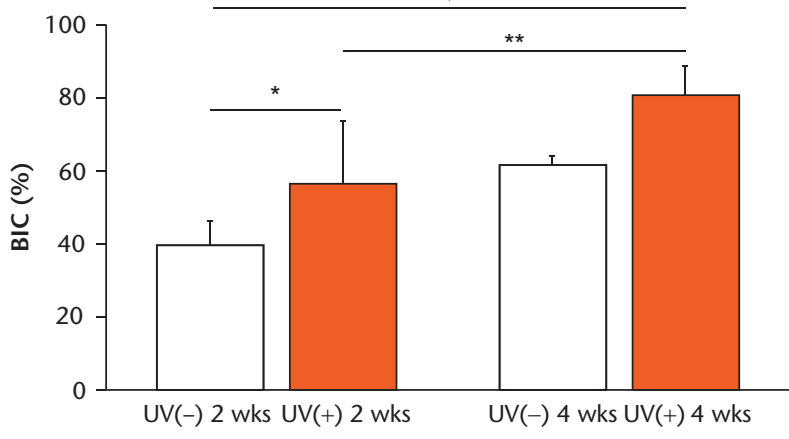

Fig. 4i
Light microscope images (magnification $\times 4$ and $\times 10$, higher magnification images of the boxed areas) two and four weeks after implantation. The micrographs show the bone response for (A, C, E, G) 64Ti(-) and (B, D, F, H) 64Ti(+) implants. Scale bar: $200 \mu \mathrm{m}$. (I) BIC ratios for $64 \mathrm{Ti}(-)$ and $64 \mathrm{Ti}(+)$ were calculated. Results are shown as mean percentage \pm standard deviation. $\mathrm{p}<0.05$, *64Ti(-) vs 64Ti(+), †64Ti(-) 2 w vs 64Ti(+) $4 \mathrm{w}(\mathrm{n}=5)$.

irradiation, whereas the amount of oxygen significantly increased after UV irradiation (Fig. 1).

Contact angle analysis. The contact angle indicated that both $\mathrm{Ti}$ and Ti6Al4V had a hydrophobic surface before UV irradiation, with a mean contact angle of $64.3^{\circ}$ and $72.3^{\circ}$, respectively. After UV irradiation, both surfaces became hydrophilic, with a contact angle of $11.1^{\circ}$ and $6.0^{\circ}$ degrees, respectively. The contact angle of both surfaces increased and the hydrophilicity decreased with time. There were significant differences between the UV(-) and UV(+) groups immediately, and at 0 and 30 minutes, one, three and six hours, and two weeks. However, there was no significant difference between the UV(-) and UV(+) groups at four weeks (Fig. 2). 


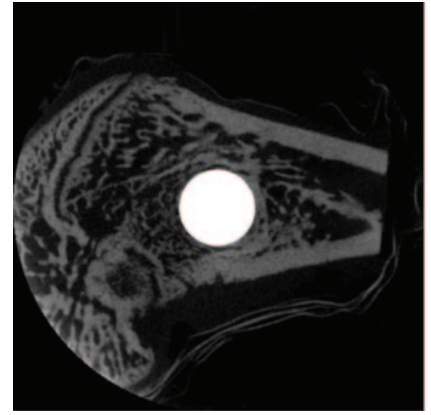

Fig. $5 a$

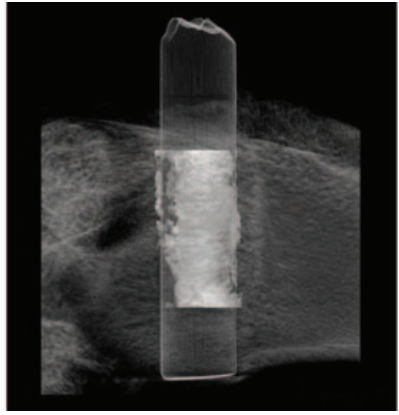

Fig. $5 b$

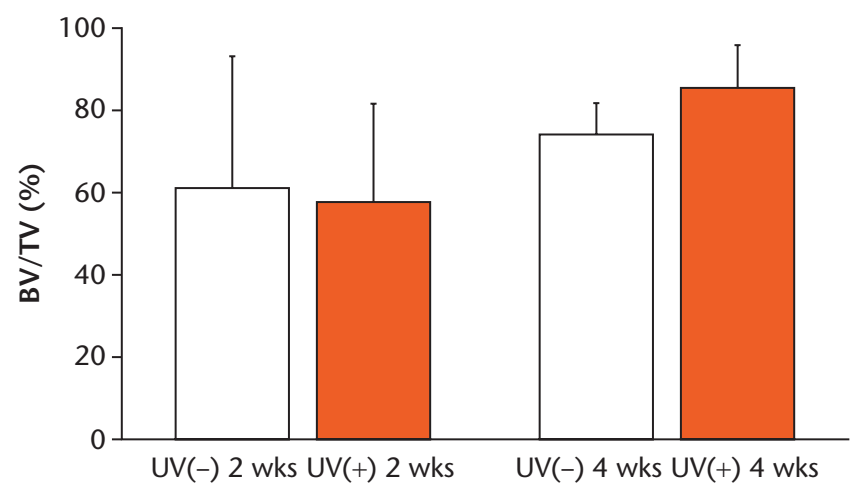

Fig. 5c

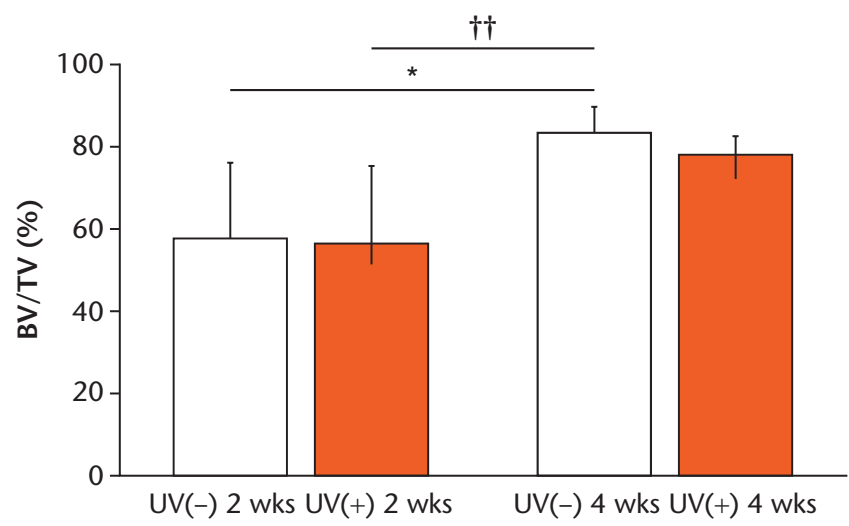

Fig. $5 d$

BV ratio was calculated using micro $\mathrm{CT}$. a) The image shows a representative cross-sectional microcomputed tomography slice in the perpendicular direction to the longitudinal axis of the implant. Scale bar: $1 \mathrm{~mm}$. b) The image shows a representative three-dimensional computed tomography image around the implant. Scale bar: $2000 \mu \mathrm{m}$. BV ratios for c) Ti and d) Ti6Al4V were calculated. Results are shown as mean percentage and standard deviation. $\mathrm{p}<0.05$, *UV(-) vs UV(+), ††64Ti(-) 2 w vs 64Ti(-) $4 \mathrm{w}(\mathrm{n}=5)$.

Histological analysis. In the $\mathrm{Ti}$ groups, the mean $\mathrm{BIC}$ ratio was $39.8 \%$ for $\mathrm{Ti}(-)$ and $56.8 \%$ for $\mathrm{Ti}(+)$ at two weeks, and $61.6 \%$ for $64 \mathrm{Ti}(-)$ and $80.7 \%$ for $64 \mathrm{Ti}(+)$ at four weeks. There was a significant difference in the $\mathrm{BIC}$ ratio between $\mathrm{Ti}(-)$ and $\mathrm{Ti}(+)$ at two weeks, but no significant difference at four weeks (Fig. 3). In the Ti6Al4V groups, the mean $\mathrm{BIC}$ ratio was $44.4 \%$ for $64 \mathrm{Ti}(-)$ and $65.0 \%$ for $64 \mathrm{Ti}(+)$ at two weeks, and $58.6 \%$ for $64 \mathrm{Ti}(-)$ and $76.3 \%$ for $64 \mathrm{Ti}(+)$ at four weeks. Similarly, there was a significant difference in the $\mathrm{BIC}$ ratio between $64 \mathrm{Ti}(-)$ and $64 \mathrm{Ti}(+)$ at two weeks, but no significant difference at four weeks (Fig. 4).

Bone formation around samples. In the control groups, the mean BV ratio was $61.6 \%$ for $\mathrm{Ti}(-)$ and $55.9 \%$ for $64 \mathrm{Ti}(-)$ at two weeks, and $74.5 \%$ for $\mathrm{Ti}(-)$ and $80.8 \%$ for $64 \mathrm{Ti}(-)$ at four weeks. In the UV-treated groups, the mean $\mathrm{BV}$ ratio was $58.0 \%$ for $\mathrm{Ti}(+)$ and $55.0 \%$ for $64 \mathrm{Ti}(+)$ at two weeks, and $86.4 \%$ for $\mathrm{Ti}(+)$ and $76.0 \%$ for $64 \mathrm{Ti}(+)$ at four weeks. There were no significant differences between $\mathrm{Ti}(-)$ and $\mathrm{Ti}(+)$ or between $64 \mathrm{Ti}(-)$ and $64 \mathrm{Ti}(+)$ at two or four weeks (Fig. 5).

\section{Discussion}

This study demonstrated that photofunctionalisation reduced the amount of carbon on $\mathrm{Ti}$ and $\mathrm{Ti} 6 \mathrm{Al} 4 \mathrm{~V}$ surfaces, and changed the surfaces from hydrophobic to hydrophilic. The BIC ratios of both surfaces at two weeks were significantly higher in the UV-treated than the control groups, however, there was no significant difference between the UV-treated and control groups at four weeks.

Some studies have demonstrated that removal of carbon deposition from a Ti surface enhances the bioactivity and osseointegration of Ti. ${ }^{16}$ Hydroxyl groups increase the wettability and hydrophilicity on the Ti surface, which enhances the initial attachment, proliferation, and differentiation of osteoblasts. ${ }^{17-19}$ The results of Ti6Al4V in this study are similar to those in previous reports. $3,5,13$ The previous studies indicated that there were no differences in surface morphology before and after UV irradiation. 3,20 This study suggests that the carbon deposition on the $\mathrm{Ti}$ and Ti6Al4V surfaces was removed after UV irradiation, increasing the hydroxyl groups and hydrophilicity on both surfaces.

In one study, photofunctionalisation of $\mathrm{Ti}$ implants increased the BIC ratio for UV-treated implants 2.5-fold at two weeks and 1.9-fold at four weeks compared with control implants. ${ }^{3}$ However, no reports have described the BIC ratio for UV-treated Ti6AI4V. In this study, the BIC ratio for UV-treated Ti6Al4V implants at two weeks was significantly higher than that of control implants, but the BIC ratio for UV-treated implants at four weeks was not significantly different. Previous studies reported that photofunctionalisation of $\mathrm{Ti}$ and Ti6Al4V surfaces enhanced attachment, spread, proliferation, and differentiation of osteoblasts in vitro.3,13 The present study suggests that photofunctionalisation enhances the bioactivity and osteoconductivity of both surfaces. Notably, the BIC ratios were similar between the UV-treated groups at two weeks and control groups at four weeks, clearly demonstrating an acceleration of osseointegration. Thus, photofunctionalisation of $\mathrm{Ti}$ and Ti6Al4V implants enhances osseointegration in the early phase. A previous study reported that after a healing period of 12 weeks, there was no difference in the BIC ratio for Ti implants between the UV-treated and control groups. ${ }^{21}$ However, when using implants for fracture operations, earlier osseointegration is important. 
The present study indicated no significant differences in the $B V$ ratio between UV-treated and control implants at two and four weeks. In one study, the BV for UV-treated implants at two weeks was almost equal to that of control implants, although UV-treated implants were stronger and more resistant to the forces of mechanical stress than control implants. ${ }^{22}$ Photofunctionalisation enhances osteogenesis around the implant, increases interfacial bone deposition on $\mathrm{Ti}$, and improves the marginal bone seal and support. ${ }^{23}$ It has been suggested that photofunctionalisation induces denser cortical bone formation and a stiffer bone connection compared with control implants. ${ }^{22}$

The study had some limitations. First, implant biomechanical tests to assess the biomechanical strength of bone-implant integration were not performed. Previous studies showed that the push-in values for photofunctionalised Ti and Ti6Al4V implants were significantly higher than those for control implants. ${ }^{3,13}$ Second, only five specimens were included in each group, making it difficult to come to a statistical conclusion. Third, to analyse periimplant bone regeneration, fluorochrome staining was not performed. This method can be used as a dynamic evaluation of bone regeneration. Further studies are needed to confirm the biomechanical strength and the fluorochrome staining evaluation of the implants used in this study.

In conclusion, the present study revealed the effect of photofunctionalisation on Ti6AI4V in vivo. As was seen by Yamamura et al ${ }^{18}$ the surface of UV-treated implants demonstrated significant differences in chemical properties and wettability. Additionally, photofunctionalisation induced an accelerated increase in the BIC ratios in the early healing stage. This technology may enable early load bearing and decrease morbidity by application to orthopaedic implants.

\section{References}

1. Liu X, Chu PK, Ding C. Surface modification of titanium, titanium alloys, and related materials for biomedical applications. Mater Sci Eng Rep 2004;47:49-121.

2. Navarro M, Michiardi A, Castaño O, Planell JA. Biomaterials in orthopaedics. J $R$ Soc Interface 2008;5:1137-1158

3. Aita H, Hori N, Takeuchi M, et al. The effect of ultraviolet functionalization of titanium on integration with bone. Biomaterials 2009;30:1015-1025.

4. Ogawa T, Nishimura I. Different bone integration profiles of turned and acid-etched implants associated with modulated expression of extracellular matrix genes. Int $\mathrm{J}$ Oral Maxillofac Implants 2003;18:200-210.

5. Att W, Hori N, Takeuchi $\mathbf{M}$, et al. Time-dependent degradation of titanium osteoconductivity: an implication of biological aging of implant materials. Biomaterials 2009;30:5352-5363.

6. Kilpadi DV, Lemons JE, Liu J, et al. Cleaning and heat-treatment effects on unalloyed titanium implant surfaces. Int J Oral Maxillofac Implants 2000;15:219-230.

7. Hori N, Att W, Ueno T, et al. Age-dependent degradation of the protein adsorption capacity of titanium. J Dent Res 2009;88:663-667.

8. Hayashi R, Ueno T, Migita S, et al. Hydrocarbon deposition attenuates osteoblast activity on titanium. J Dent Res 2014;93:698-703.

9. Wei J, Yoshinari M, Takemoto S, et al. Adhesion of mouse fibroblasts on hexamethyldisiloxane surfaces with wide range of wettability. J Biomed Mater Res B Appl Biomater 2007;81:66-75
10. Altankov G, Grinnell F, Groth T. Studies on the biocompatibility of materials: fibroblast reorganization of substratum-bound fibronectin on surfaces varying in wettability. J Biomed Mater Res 1996;30:385-391.

11. Groth T, Altankov G. Studies on cell-biomaterial interaction: role of tyrosine phosphorylation during fibroblast spreading on surfaces varying in wettability. Biomaterials 1996;17:1227-1234.

12. Funato A, Yamada M, Ogawa T. Success rate, healing time, and implant stability of photofunctionalized dental implants. Int J Oral Maxillofac Implants 2013;28:1261-1271.

13. Minamikawa H, lkeda T, Att W, et al. Photofunctionalization increases the bioactivity and osteoconductivity of the titanium alloy Ti6Al4V. J Biomed Mater Res A 2014; 102:3618-3630.

14. Yang $\mathbf{M}$, Lin $\mathbf{S}$. A method for correcting the contact angle from the $\mathrm{u} / 2$ method. Colloids Surf A Physicochem Eng Asp 2003;220:199-210.

15. Yamazaki M, Yamada M, Ishizaki K, Sakurai K. Ultraviolet-C irradiation to titanium implants increases peri-implant bone formation without impeding mineralization in a rabbit femur model. Acta Odontol Scand 2015;73:302-311.

16. Hayashi R, Ueno T, Migita S, et al. Hydrocarbon deposition attenuates osteoblast activity on titanium. J Dent Res 2014;93:698-703.

17. Arima Y, Iwata H. Effect of wettability and surface functional groups on protein adsorption and cell adhesion using well-defined mixed self-assembled monolayers. Biomaterials 2007;28:3074-3082.

18. Yamamura K, Miura T, Kou I, et al. Influence of various superhydrophilic treatments of titanium on the initial attachment, proliferation, and differentiation of osteoblastlike cells. Dent Mater J 2015;34:120-127.

19. Sartoretto SC, Alves AT, Resende RF, et al. Early osseointegration driven by the surface chemistry and wettability of dental implants. J Appl Oral Sci 2015;23: 279-287.

20. Sawase T, Jimbo R, Baba K, et al. Photo-induced hydrophilicity enhances initial cell behavior and early bone apposition. Clin Oral Implants Res 2008;19:491-496.

21. Hayashi M, Jimbo R, Xue Y, et al. Photocatalytically induced hydrophilicity influences bone remodelling at longer healing periods: a rabbit study. Clin Oral Implants Res 2014;25:749-754.

22. Hirota $\mathbf{M}$, Tanaka $\mathbf{M}$, Ishijima $\mathbf{M}$, et al. Effect of photofunctionalization on Ti6Al4V screw stability placed in segmental bone defects in rat femurs. J Oral Maxillofac Surg 2016;74:861.e1-861.e16

23. Pyo SW, Park YB, Moon HS, Lee JH, Ogawa T. Photofunctionalization enhances bone-implant contact, dynamics of interfacial osteogenesis, marginal bone seal, and removal torque value of implants: a dog jawbone study. Implant Dent 2013;22:666-675.

\section{Funding Statement}

- This research received no specific grant from any funding agency in the public, commercial or not-for-profit sectors.

Author Contribution

- R. Yamauchi: Conceived the study concept, study design, animal care and surgery, $\mathrm{X}$-ray photoelectron spectroscopy analysis, contact angle analysis, and histological analysis and supported this study, wrote the manuscript, participated in interpretation of the results and approved the final version.

- T. Itabashi: Conceived the study concept, study design, animal care and surgery, $X$-ray photoelectron spectroscopy analysis, contact angle analysis, and histological analysis and supported this study, wrote the manuscript, participated in interpretation of the results and approved the final version.

- K. Wada: Conceived the study concept, study design, X-ray photoelectron spectroscopy analysis, contact angle analysis, and histological analysis and supported this study, participated in interpretation of the results and approved the final version. study, participated in interpretation of the results and approved the final version.
T. Tanaka: Conceived the study concept, study design, X-ray photoelectron spectroscopy analysis, contact angle analysis, and histological analysis and supported this study, participated in interpretation of the results and approved the final version.

- G. Kumagai: Conceived the study concept, study design, X-ray photoelectron spectroscopy analysis, contact angle analysis, and histological analysis and supported this study, wrote the manuscript, participated in interpretation of the results and approved the final version.

Y. Ishibashi: Conceived the study concept, study design, provided study materials
Y a proved the final verion. and supported this study, supervised the whole project and wrote the manuscript, participated in interpretation of the results and approved the final version.

ICMJE Conflicts of Interest

None declared

(c) 2017 Yamouchi et al. This is an open-access article distributed under the terms of the Creative Commons Attributions licence (CC-BY-NC), which permits unrestricted use, distribution, and reproduction in any medium, but not for commercial gain, provided the original author and source are credited. 